\title{
Oligodendroglioma cells shed microvesicles which contain TRAIL as well as molecular chaperones and induce cell death in astrocytes
}

\author{
ALESSANDRA LO CICERO $^{1}$, GABRIELLA SCHIERA ${ }^{1}$, PATRIZIA PROIA ${ }^{2}$, PATRIZIA SALADINO ${ }^{3}$, \\ GIOVANNI SAVETTIERI ${ }^{1}$, CARLO MARIA DI LIEGRO ${ }^{3}$ and ITALIA DI LIEGRO ${ }^{1}$ \\ ${ }^{1}$ Dipartimento di Biomedicina Sperimentale e Neuroscienze Cliniche, ${ }^{2}$ Dipartimento di Studi Giuridici, Economici, \\ Biomedici, Psicosociopedagogici delle Scienze Motorie e Sportive, and ${ }^{3}$ Dipartimento di Scienze e Tecnologie \\ Molecolari e Biomolecolari (STEMBIO), Università degli Studi di Palermo, Palermo, Italy
}

Received June 9, 2011; Accepted July 12, 2011

DOI: $10.3892 /$ ijo.2011.1160

\begin{abstract}
Microvesicles (MVs) shed from G26/24 oligodendroglioma cells were previously reported to cause a reproducible, dose-dependent, inhibitory effect on neurite outgrowth, and eventually neuronal apoptosis, when added to primary cultures of rat cortical neurons. These effects were reduced but not abolished by functional monoclonal antibodies against Fas-L. In order to investigate whether MVs contain other factors able to induce cell death, we tested them for TRAIL and found clear evidence of its presence in the vesicles. This finding suggests the possibility that Fas-L and TRAIL cooperate in inducing brain cell death. Aimed at understanding the route through which the vesicles deliver their messages to the target cells, we labeled oligodendroglioma cells with radioactive methionine and then added the labeled vesicles shed from tumor cells to unlabeled astrocytes in culture. Here we report that labeled proteins were delivered to the test cells. In order to investigate whether astrocytes, like neurons, are sensitive to oligodendroglioma-derived vesicles, MVs were prepared from media conditioned by G26/24 oligodendroglioma cells and added to primary cultures of rat cortical astrocytes. These cells were clearly more resistant than neurons to microvesicle-induced damage: a high dose (40 $\mu \mathrm{g})$ of shed MVs induced cell death in only about $40 \%$ of astrocytes. Finally, we demonstrated that Hsp70 is specifically enriched in MVs which also contain, even if at lower level, the Hsc70 constitutive chaperone.
\end{abstract}

\section{Introduction}

A peculiar route for cell-to-cell communication could be mediated by extracellular membrane vesicles. Two distinct types of signaling vesicles have been identified up to now: i) vesicles with a diameter of 30-100 nm, known as exosomes, released from

Correspondence to: Professor Italia Di Liegro, Dipartimento di Biomedicina Sperimentale e Neuroscienze Cliniche, Sezione di Scienze Biochimiche, Via del Vespro 129, I-90127 Palermo, Italy

E-mail: diliegro@unipa.it

Key words: microvesicles, oligodendroglioma, astrocytes, TRAIL, Hsp70 the cell by exocytosis of multivesicular bodies (MVBs), intracellular organelles of the endosomal system; ii) small vesicles, called shed vesicles (SVs), $100 \mathrm{~nm}-1 \mu \mathrm{m}$ in diameter, released into the extracellular space by direct budding from the plasma membrane. Mixed vesicle populations, containing both shedding vesicles and exosomes, will be referred to as microvesicles (MVs) (1,2).

Upon release, both types of microvesicles circulate in the extracellular space adjacent to the site of discharge where they can be broken down, often within a few minutes. Some of them, however, can move some distance by diffusion and can appear in biological fluids, such as cerebrospinal fluid, blood and urine. In some cases microvesicles deliver transmembrane signals which activate surface receptors (3); in other cases they function not only as messengers, but also as platforms necessary for the coordinate development of multisignaling processes (4). Being membrane-bound structures, microvesicles can fuse with their target cells. Because of these properties, MVs, which seem to be enriched in specific proteins and mRNAs (5), have been suggested to function in signaling as well as in the horizontal transfer of their membrane and/or cargo molecules (6).

Different neural cells release MVs into the extracellular space. Neurons and astrocytes shed extracellular vesicles which contain FGF2 and VEGF and could be involved in interaction with endothelial cells, to form the blood-brain barrier $(7,8)$. Also brain tumor cells release MVs in the extracellular space. Glioblastomas release exosomes containing mRNA, miRNA and angiogenic proteins (9).

Previous studies on oligodendroglioma cell cultures revealed that these cells shed MVs which inhibited neurite sprouting and caused apoptosis to primary fetal rat neurons, after $48-72 \mathrm{~h}$ of incubation (10). Proapoptotic effects of vesicles on neurons were dose-dependent and were induced also when concentrations of tumor-derived vesicles were similar to those probably occurring in vivo, when an oligodendroglioma tumor is growing inside the central nervous system (CNS).

These vesicles contain Fas-L, a molecule that has been identified also in vesicles shed by other kinds of tumor cells $(11,12)$, while, on the other hand, Fas receptor has been reported to be expressed both in neurons and astrocytes (13). The presence of Fas- $\mathrm{L}$ in vesicles shed by tumor cells is generally considered as part of the mechanism by which tumors acquire the capability 
to escape from host immune surveillance. The presence of the molecule in vesicles shed by oligodendroglioma cells appears to have an additional meaning in allowing production of a cell-free environment in which tumor cells can grow. Interestingly, functional monoclonal antibodies against Fas-L were able to reduce but not to abolish the inhibitory effects of vesicles on neurite growth and their proapoptotic effects on neurons. In looking for additional factors involved in cell death, in the present study we investigated the possible presence of TRAIL in MVs. We also analyzed loading of MVs with molecular chaperones. Finally, we studied the effects of G26/24-shed MVs on astrocytes.

\section{Materials and methods}

Animals. Procedures involving animals were conducted according to the European Community Council Directive 86/609, OJL 358 1, December 12, 1987. Winstar rats (Stefano Morini, San Polo d'Enza, Italy) were housed in the institutional animal care facility of Dipartimento di Scienze e Tecnologie Molecolari e Biomolecolari (STEMBIO), University of Palermo, Palermo, Italy, under the direction of a licensed veterinarian who approved the protocols.

Cell culture. Astrocytes were purified from brain cortices of 2-day-old rats, as described by Cole and De Vellis (14), exploiting the differences in adhesion of the various cell types.

G26/24 oligodendroglioma cells and primary astrocytes were cultured in DME-Ham's F-12 (2:1) medium supplemented with $10 \%$ fetal calf serum (FCS), and 100,000 U penicillin, $100 \mathrm{mg}$ streptomycin and $250 \mu \mathrm{g}$ amphotericin B per liter. Cell cultures were maintained in humidified $5 \% \mathrm{CO}_{2} / 95 \%$ air, at $37^{\circ} \mathrm{C}$.

Preparation of microvesicles from the cell culture medium. Vesicles were prepared from oligodendroglioma G26/24 subconfluent healthy cells grown in FCS-free medium, as previously described (15). After $24 \mathrm{~h}$ of culture, conditioned media were centrifuged at $2,000 \mathrm{x}$ g for $15 \mathrm{~min}$ and then at 4,000 $\mathrm{x} \mathrm{g}$ for $15 \mathrm{~min}$. The supernatant was centrifuged at 105,000 x g (Ti60 Rotor, Beckman) for $90 \mathrm{~min}$ at $4^{\circ} \mathrm{C}$. Pelleted vesicles were suspended with phosphate-buffered saline, $\mathrm{pH} 7.5$ (PBS) and the amount of isolated vesicles was determined by measuring protein concentration by the Bradford microassay method (BioRad) using bovine serum albumin (Sigma-Aldrich) as a standard.

Cell lysate preparation. Cultured cells were washed in PBS, scraped from the wells, and centrifuged at $600 \mathrm{x} \mathrm{g}$ for $5 \mathrm{~min}$. The pellet was homogenized in homogenization buffer $(0.32 \mathrm{M}$ sucrose; $50 \mathrm{mM}$ sodium phosphate buffer, $\mathrm{pH} 6.5 ; 50 \mathrm{mM} \mathrm{KCl}$; $0.5 \mathrm{mM}$ spermine; $0.15 \mathrm{mM}$ spermidine; $2 \mathrm{mM}$ EDTA; and $0.15 \mathrm{mM}$ EGTA), containing the protease inhibitors aprotinin $(2 \mu \mathrm{g} / \mathrm{ml})$, antipain $(2 \mu \mathrm{g} / \mathrm{ml})$, leupeptin $(2 \mu \mathrm{g} / \mathrm{ml})$, pepstatin A (2 $\mu \mathrm{g} / \mathrm{ml})$, benzamidine $(1.0 \mathrm{mM})$, and phenylmethylsulfonyl fluoride (PMSF) (1.0 mM), all from Sigma-Aldrich (MO, USA).

Western blot analyses. After electrophoresis (SDS-PAGE), total proteins were immunoblotted onto a PVDF (Polyvinylidene fluoride) membrane (Immobilon P, Millipore, MA, USA). The gel was equilibrated with CAPS $1 \mathrm{X}(100 \mathrm{mM}$ $\mathrm{N}$-cyclohexyl-3-aminopropanesulfonic acid, $\mathrm{pH} 11.0+10 \%$ methanol). The PVDF membrane was activated by wetting in methanol $100 \%$ for $30 \mathrm{sec}$, and then equilibrated with CAPS $1 \mathrm{X}$ for $30 \mathrm{sec}$. The immunoblot was done in the Trans-Blot ${ }^{\circledR}$ Bior-Rad for $45 \mathrm{~min}$ at $170 \mathrm{~mA}$. After blotting, the membrane, that contains the proteins, was saturated with $3 \% \mathrm{BSA}, 10 \%$ FCS, PBS $1 \mathrm{X}$ for $1 \mathrm{~h}$, and then incubated overnight with one of the following antibodies: anti-TRAIL, anti-Hsp70, anti-Hsp90, anti-Hsc70. The antibodies are in 3\% BSA, 1X PBS.

After washing with PBS containing 0.1\% Tween-20, the membranes were incubated for $1 \mathrm{~h}$ with alkaline phosphataseconjugated secondary antibodies. Immunocomplexes were visualized by adding the substrates of alkaline phosphatase, NBT and BCIP (Sigma-Aldrich).

Treatments of astrocytes with vesicles and vitality assay. Effects of vesicles shed from G26/24 cells into the medium were analyzed by adding to primary cultures of astrocytes an aliquot of MVs containing 10-40 $\mu \mathrm{g}$ of proteins. The effects were analyzed after $48 \mathrm{~h}$ of culture by vitality assay: cells were stained with a combination of the fluorescent DNA-binding dyes, acridine orange (AO) and ethidium bromide (EB), $100 \mu \mathrm{g} / \mathrm{ml}$ in PBS for each dye (AO/EB colorimetric assay). The differential uptake of these two dyes allowed identification of viable and non-viable cells by fluorescence microscopy: normal nuclei in alive cells appeared bright green; apoptotic nuclei in dead cells appeared bright orange with highly condensed chromatin. Statistical analysis of the results was done by counting green and orange/red cells in different fields. Numbers obtained from different experiments were used to calculate average percentages of viable and dead cells, as well as standard deviations.

Metabolic labeling of $\mathrm{G} 26 / 24$ cells and astrocytes with ${ }^{35} \mathrm{~S}$ methionine. G26/24 cells and astrocytes were incubated for $12 \mathrm{~h}$ in a methionine-free medium, to make them proner to methionine uptake afterward. Then cells were grown in methionine-free medium, to which $4 \mu \mathrm{Ci}$ of ${ }^{35} \mathrm{~S}$-methionine were added/ml of medium, and cultured for $16 \mathrm{~h}$. During this period, they started producing labeled proteins. Cells were washed once with PBS and then cultured for $24 \mathrm{~h}$ in NIH serum-free medium. Medium conditioned by G26/24 cells was finally collected and centrifuged to obtain labeled vesicles. When the last conditioned medium was collected, cells were also lysed to obtain total labeled cell proteins. From astrocytes, total labeled cell lysates were prepared directly. Total cell lysates as well as G26/24-derived MV proteins were analyzed by SDS-PAGE and fluorography.

Treatments of astrocytes with ${ }^{35}$ S-labeled vesicles. Primary cultures of astrocytes were incubated for $24 \mathrm{~h}$ with ${ }^{35} \mathrm{~S}$-labeled vesicles from G26/24 cells, and then washed with PBS. Cells were lysed to obtain total cell proteins. Total proteins from control labeled astrocytes, from astrocytes pre-incubated with labeled MVs shed by G26/24 cells, and from labeled MVs were analyzed by SDS-PAGE and fluorography.

\section{Results}

Extracellular vesicles interact with target cells. To clarify the fate of MVs after shedding into the extracellular space and their interaction with target cells, G26/24 cells and primary astro- 


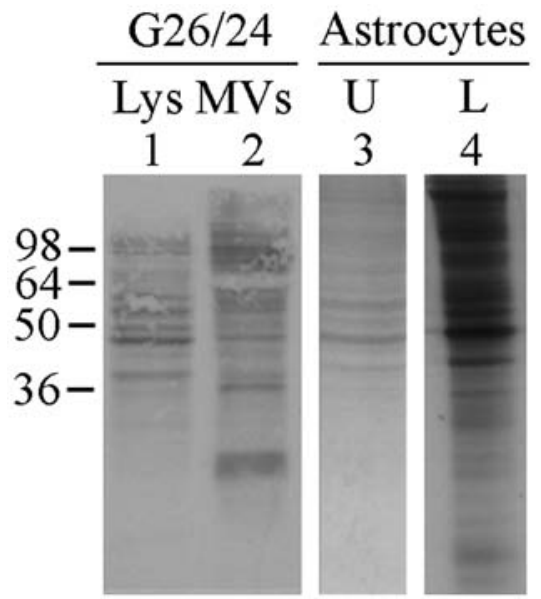

Figure 1. SDS-PAGE and fluorography of G26/24 total lysates (lane 1, Lys) and shed microvesicles (lane 2, MVs) as well as of unlabeled astrocytes (lane 3, U) incubated with ${ }^{35} \mathrm{~S}$-labeled vesicles shed from G26/24 cells. Total cell lysates from labeled astrocytes were also analyzed as a control (lane $4, \mathrm{~L}$ ).

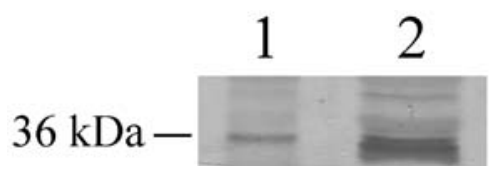

Figure 2. Microvesicles contain TRAIL. Western blot analysis of microvesicles (lane 1) and total lysates from G26/24 cells (lane 2). Proteins were immunostained with an anti-TRAIL antibody.

cytes were metabolically labeled with ${ }^{35} \mathrm{~S}$-methionine. If labeled proteins entered MVs, they could be used to trace the destiny of MV components after release from G26/24 cells. From both labeled G26/24 cells (Fig. 1, lane 1: Lys) and astrocytes (Fig. 1, lane 4: L) total cell lysates were also prepared, in order to have reference patterns of total labeled proteins from the two cell types. In order to study their fate, labeled MVs (Fig. 1, lane 2: MVs) were added to unlabeled astrocytes (Fig. 1, lane 3: U). In detail, astrocytes were incubated for $24 \mathrm{~h}$ with MVs containing 10-40 $\mu \mathrm{g}$ of proteins, and then washed with PBS containing an excess of unlabeled methionine to eliminate traces of labeled material before homogenizing the cells. SDS-PAGE analysis of lysates, followed by fluorography of the gel revealed the presence of labeled bands in unlabeled astrocytes (Fig. 1, lane 3: U), suggesting transfer of labeled proteins, probably through fusion of MVs with the plasma membrane of unlabeled target cells.

Extracellular vesicles shed from G26/24 cells contain TRAIL and induce astrocyte damage. Oligodendroglioma G26/24 cells are already known to release into the culture medium MVs, which are able to induce cell death in primary neurons (10). Investigation of the composition of MVs had already evidenced the presence of Fas-L, a proapoptotic factor. However, inhibition of Fas-L activity reduced but not eliminated the apoptotic effects on neurons. Thus, we investigated possible presence of other factors, besides Fas-L, in MVs. A likely candidate seemed tumor necrosis factor-related apoptosis-inducing ligand (TRAIL), a protein with well known pro-apoptotic activity $(16,17)$. We found that TRAIL is visible both in microvesicles (lane 1) and total cell lysates (lane 2) from G26/24 oligodendroglioma cells (Fig. 2).

The effects of purified MVs were directly tested on astrocytes. Astrocytes were treated for $48 \mathrm{~h}$ with aliquots of MVs containing 10-40 $\mu \mathrm{g}$ of proteins, and cell vitality was assayed by acridine orange and ethidium bromide staining. Astrocytes were quite resistant to the lowest doses of vesicles (not shown). When treated with $40 \mu \mathrm{g}$ of proteins, however, cell death was induced on average by $\sim 25 \%$ of astrocytes (the maximum value observed was $\sim 50 \%$ ). These data suggest that oligodendroglioma-shed vesicles have cell death-inducing effects also on astrocytes, but that these cells are more resistant than neurons (10) to their effects (Fig. 3).
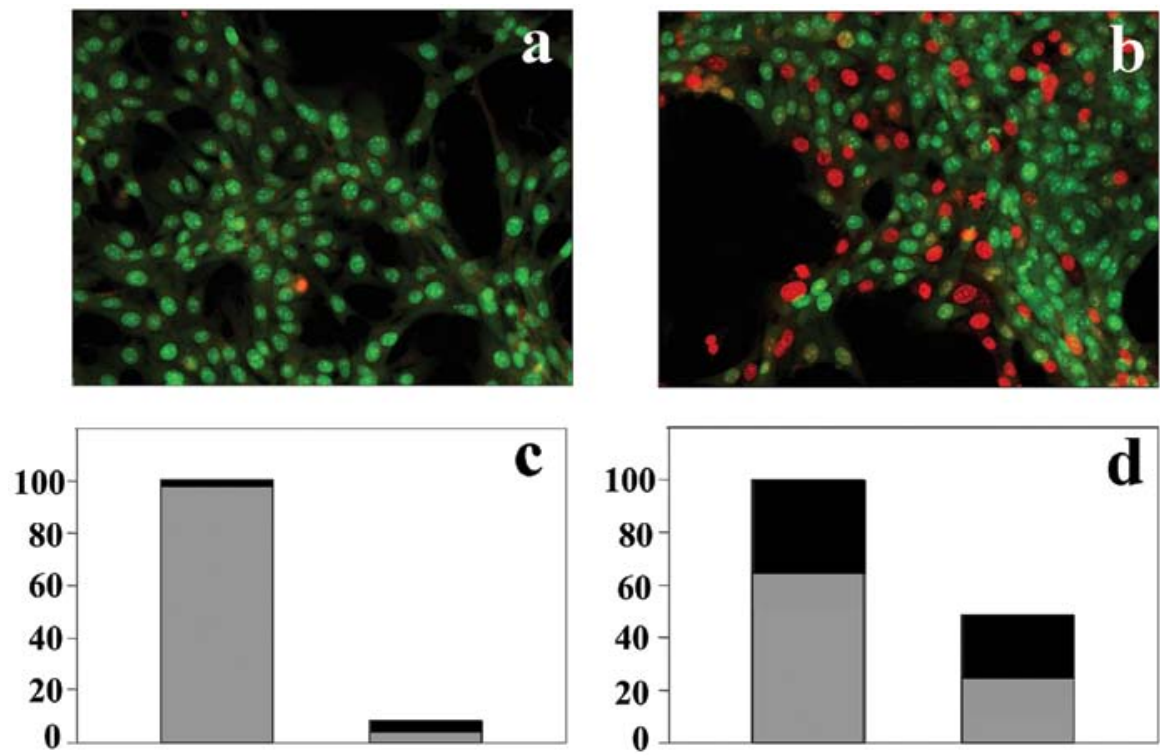

Figure 3. Effects of vesicles shed from G26/24 cells on primary cultures of astrocytes. Vitality assays with AO/EB: (a), control astrocytes; (b), astrocytes incubated with MVs from G26/24 cells for 48 h. Statistical analyses are shown in graphic form in the lower part of the figure: (c), control astrocytes; (d), astrocytes incubated with MVs. The grey bars represent means of 4 different experiments. Standard deviations are also shown (black bars). The difference between the means were highly significant $(\mathrm{p}<0.005)$. 


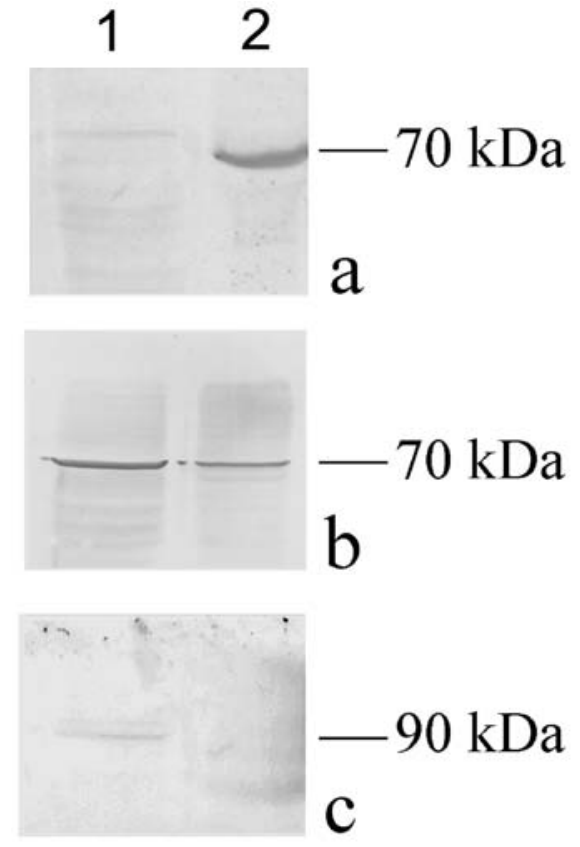

Figure 4. Vesicles shed from G26/24 cells contain Hsp proteins. Western blot analysis of G26/24 cell lysate (lane 1) and vesicles (lane 2). Proteins were immuno-stained with anti-Hsp70 (a), anti-Hsc70 (b) and anti-Hsp90 antibodies (c).

Oligodendroglioma G26/24 cells release Hsp70 and Hsc 70 in the extracellular culture medium. MVs shed from G26/24 cells were finally tested by Western blotting for the presence of molecular chaperones. The results of these assays showed that Hsp70 chaperone is highly enriched in MVs respect to the whole cell lysate, where it is not visible at all (Fig. 4).

As a control we also studied distribution of $\mathrm{Hsc70}$ and Hsp90. The first protein is present both in MVs and in the total cell lysate, whereas Hsp90 is only visible in the cell lysate, at a very low level.

\section{Discussion}

Oligodendrogliomas are slowly growing tumors which however cause brain damage. This effect has been primarily attributed to compression on surrounding brain cells, caused by the increasing size of the tumor. More recently, it was suggested that G26/24 oligodendroglioma cells can actively induce neuronal damage by releasing molecules able to inhibit neurite sprouting and to eventually cause neuronal death (10). Interestingly, such molecules seem to be released through an unusual route involving shedding of extracellular membrane microvesicles (10).

MVs originate from the plasma membrane through a mechanism which resembles budding of viruses from infected cells and requires production of RNA and proteins. Although tumor cells are particularly active in shedding MVs, it has been reported that vesicles are also released from non-tumor cells $(7,8,18,19)$. On the other hand, it remains not completely understood how microvesicles deliver their cargoes and whether or not they fuse with their target cells. Our results confirmed the hypothesis that at least part of the vesicle population released from oligodendroglioma cells fuse with surrounding cells. Labeled proteins were indeed found in lysates from unlabeled astrocytes incubated with labeled microvesicles shed by G26/24 cells.

Previous analyses demonstrated that extracellular microvesicles (MVs) released from G26/24 cells contain Fas-L, a well known pro-apoptotic agent (10). Moreover, functional monoclonal antibodies against Fas-L were able to counteract at least in part the pro-apoptotic effect of MVs (10).

The fact that the counteracting effect of anti-Fas-L antibodies was only partial suggested that other cell death-inducing molecules are present in MVs. In looking for other pro-apoptotic proteins present in the microvesicles, we tested the vesicle fraction for TRAIL and found clear evidence of its presence in MVs. This finding suggested a possible involvement of TRAIL, in synergy with Fas-L, in the pro-apoptotic effects of vesicles on brain cells.

To understand the role of MVs in apoptosis and their possible interaction with different brain cell types, we investigated their effects on primary astrocytes, which have been reported to be resistant to different stresses which are more effective on neurons. As shown in the present study, 25-50\% of astrocytes were damaged when incubated with $40 \mu \mathrm{g}$ of shed MVs. However, they were almost unaffected (data not shown) when treated with lower doses of vesicles, already able to kill neurons (10).

Heat shock protein 70 (Hsp70) is a chaperone known to be involved in the formation of both intracellular and extracellular vesicular structures, such as those related to viral budding. Specialized membrane microdomains, known as lipid rafts, may play a role in Hsp70 exocytosis (20), inducing the release of the protein in the extracellular space in response to cellular stress. Moreover, Hsp70 seemed to be involved in an exosome-dependent trafficking (21). It has been also suggested that extracellular Hsp70 may act as a potent danger signal to the immune system (22). Here we demonstrated that Hsp70 is specifically enriched in the microvesicles which also contain, even if at much lower level, the Hsc70 constitutive chaperone. On the other hand, we did not find evidence for the presence in MVs of the Hsp90 chaperone.

\section{Acknowledgments}

This work was supported by a special grant of Merck Serono S.p.A. to G. Savettieri and by the University of Palermo (Università degli Studi di Palermo). A. Lo Cicero was supported by a PhD fellowship of the University of Palermo, Italy. G. Schiera was supported by a post-doctoral fellowship from the same University.

\section{References}

1. Muralidharan-Chari V, Clancy J, Plou C, Romao M, Chavrier P, Raposo G and D'Souza-Schorey C: ARF6-regulated shedding of tumor cell-derived plasma membrane microvesicles. Curr Biol 19: $1875-1885,2009$.

2. Mathivanan S, Ji H and Simpson RJ: Exosomes: extracellular organelles important in intercellular communication. J Proteomics 73: $1907-1920,2010$

3. Bianco F, Pravettoni E, Colombo A, Schenk U, MöllerT, Matteoli M and Verderio C: Astrocyte-derived ATP induces vesicle shedding and IL-1 beta release from microglia. J Immunol 174: 7268-7277, 2005.

4. Del Conde I, Shrimpton CN, Thiagarajan P and López JA: Tissue-factor-bearing microvesicles arise from lipid rafts and fuse with activated platelets to initiate coagulation. Blood 106: 1604-1611, 2005. 
5. Deregibus MC, Cantaluppi V, Calogero R, Lo Iacono M, Tetta C, Biancone L, Bruno S, Bussolati B and Camussi G: Endothelial progenitor cell derived microvesicles activate an angiogenic program in endothelial cells by a horizontal transfer of mRNA. Blood 110: 2440-2448, 2007.

6. Antonyak MA, Li B, Boroughs LK, Johnson JL, Druso JE, Bryant KL, Holowka DA and Cerione RA: Cancer cell-derived microvesicles induce transformation by transferring tissue transglutaminase and fibronectin to recipient cells. Proc Natl Acad Sci USA 108: 4852-4857, 2011.

7. Schiera G, Proia P, Alberti C, Mineo M, Savettieri G and Di Liegro I: Neurons produce FGF2 and VEGF and secrete them at least in part by shedding extracellular vesicles. J Cell Mol Med 11: 1384-1394, 2007.

8. Proia P, Schiera G, Mineo M, Ingrassia MR, Santoro G, Savettieri G and Di Liegro I: Astrocytes shed extracellular vesicles that contain fibroblast growth factor-2 and vascular endothelial growth factor. Int J Mol Med 21: 63-67, 2008

9. Skog J, Würdinger T, van Rijn S, Meijer DH, Gainche L, Sena-Esteves M, Curry WT Jr, Carter BS, Krichevsky AM and Breakefield XO: Glioblastoma microvesicles transport RNA and proteins that promote tumour growth and provide diagnostic biomarkers. Nat Cell Biol 10: 1470-1476, 2008.

10. D'Agostino S, Salamone M, Di Liegro I and Vittorelli ML: Membrane vesicles shed by oligodendroglioma cells induce neuronal apoptosis. Int J Oncol 29: 1075-1085, 2006.

11. Albanese J, Meterissian S, Kontogiannea M, Dubreuil C, Hand A, Sorba S and Dainiak N: Biologically active Fas antigen and its cognate ligand are expressed on plasma membrane-derived extracellular vesicles. Blood 91: 3862-3874, 1998.

12. Abrahams VM, Straszewski SL, Kamsteeg M, Hanczaruk B and Schwartz PE: Epithelial ovarian cancer cells secrete functional Fas ligand. Cancer Res 63: 5573-5581, 2003.

13. Choi $\mathrm{C}$ and Benveniste EN: Fas ligand/Fas system in the brain: regulator of immune and apoptotic responses. Brain Res Rev 44: $65-81,2004$.
14. Cole R and De Vellis J: Preparation of astrocytes and oligodendrocyte cultures from primary rat glial cultures. In: A Dissection and Tissue Culture Manual of the Nervous System. Alan R (ed). Liss Inc, New York, NY, pp121-133, 1989.

15. Dolo V, Ginestra A, Ghersi G, Nagase H and Vittorelli ML: Human breast carcinoma cells cultured in the presence of serum shed membrane vesicles rich in gelatinolytic activities. J Submicrosc Cytol Pathol 26: 173-180, 1994

16. Pitti RM, Marsters SA, Ruppert S, Donahue CJ, Moore A and Ashkenazi A: Induction of apoptosis by Apo-2 ligand, a new member of the tumor necrosis factor cytokine family. J Biol Chem 271: 12687-12690, 1996.

17. Dorr J, Bechmann I, Waiczies S, Aktas O, Walczak H, Krammer PH, Nitsch R and Zipp F: Lack of tumor necrosis factor-related apoptosis-inducing ligand but presence of its receptors in the human brain. J Neurosci 22: RC209, 2002.

18. Trams EG, Lauter CJ, Salem N Jr and Heine U: Exfoliation of membrane ecto-enzymes in the form of micro-vesicles. Biochem Biophys Acta 645: 63-70, 1981

19. Heijnen HF, Schiel AE, Fijnheer R, Geuze HJ and Sixma JJ: Activated platelets release two types of membrane vesicles: microvesicles by surface shedding and exosomes derived from exocytosis of multivesicular bodies and alpha-granules. Blood 94: 3791-3799, 1999.

20. Broquet AH, Thomas G, Masliah J, Trugnan G and Bachelet M: Expression of the molecular chaperone Hsp70 in detergentresistant microdomains correlates with its membrane delivery and release. J Biol Chem 278: 21601-21606, 2003.

21. Lancaster GI and Febbraio MA: Exosome-dependent trafficking of HSP70: a novel secretory pathway for cellular stress proteins. J Biol Chem 280: 23349-23355, 2005.

22. Vabulas RM, Ahmad-Nejad P, Ghose S, Kirschning CJ, Issels RD and Wagner H: HSP70 as endogenous stimulus of the Toll/interleukin-1 receptor signal pathway. J Biol Chem 277: 15107-15112, 2002 . 